Running head: THE N-PACT OF JOURNALS IN CLINICAL PSYCHOLOGY

\title{
An N-Pact Factor for Clinical Psychological Research
}

Kathleen. W. Reardon, Avanté J. Smack, Kathrin Herzhoff, \& Jennifer L. Tackett Northwestern University

Author Note

Kathleen W. Reardon, Department of Psychology, Northwestern University; Kathrin Herzhoff, Department of Psychology, Northwestern University; Avanté J. Smack, Department of Psychology, Northwestern University; Jennifer L. Tackett, Department of Psychology, Northwestern University.

This research was not supported by grant funding, nor has any portion of the data been previously analyzed or interpreted. A summary of the work was presented as a talk by the last author at the Society for Research in Psychopathology annual meeting in September 2018. A preprint version of this manuscript has been posted on PsyArXiv (psyarxiv.com/4fybk).

Correspondence concerning this article should be addressed to Kathleen W. Reardon or Jennifer L. Tackett, Department of Psychology, Northwestern University, Evanston, IL, 60208. Contact: kwreardon@u.northwestern.edu or jennifer.tackett@northwestern.edu, tel: 847-4677348 


\begin{abstract}
Although an emphasis on adequate sample size and statistical power has a long history in clinical psychological science (Cohen, 1992), increased attention to the replicability of scientific findings has again turned attention to the importance of statistical power (Bakker, van Dijk, \& Wicherts, 2012). These recent efforts have not yet circled back to modern clinical psychological research, despite the continued importance of sample size and power in producing a credible body of evidence. As one step in this process of scientific self-examination, the present study estimated an $N$-pact Factor (the statistical power of published empirical studies to detect typical effect sizes; Fraley \& Vazire, 2014) in two leading clinical journals (the Journal of Abnormal Psychology; JAP, and the Journal of Consulting and Clinical Psychology; JCCP) for the years 2000, 2005, 2010, and 2015. Study sample size, as one proxy for statistical power, is a useful focus because it allows direct comparisons with other subfields and may highlight some of the core methodological differences between clinical and other areas (e.g., hard-to-reach populations, greater emphasis on correlational designs). We found that, across all years examined, the average median sample size in clinical research is 179 participants (175 for JAP and 182 for JCCP). The power to detect a small-medium effect size of .20 is just below $80 \%$ for both journals. Although the clinical N-pact factor was higher than that estimated for social psychology, the statistical power in clinical journals is still limited to detect many effects of interest to clinical psychologists, with little evidence of improvement in sample sizes over time.

Keywords: power, sample size, replicability, clinical psychology, meta-science General Scientific Summary: Statistical power is a key indicator of whether a body of research is trustworthy. This study evaluated the median sample size of published research in two clinical psychology journals. Overall, the average median sample size for clinical psychology was greater than that calculated for social psychology, but the power in clinical journals is still limited.
\end{abstract}




\section{An N-Pact Factor for Clinical Psychological Research}

Over the past several years, psychological science has begun to engage in serious selfexamination with the aim of creating a more rigorous and trustworthy body of evidence. Although the dangers of inadequate statistical power have been discussed by psychologists for decades (Cohen, 1962; Rossi, 1990), this self-examination has led to a renewed focus on bolstering study power through larger samples as a method of protecting against false positive findings (e.g., Ioannidis, 2005; Szucs \& Ioannidis, 2017). Typical power in social and personality psychology has been described by Fraley and Vazire's (2014) N-Pact Factor (NF), a measure of the median sample size of published empirical studies. Typical statistical power in clinical psychology, however, has not yet been examined. In fact, clinical psychology has largely been left out of the ongoing conversation about improving the science (see Tackett et al., 2017), and faces some unique obstacles directly relevant to calls for increased sample size. Thus, this paper represents an extension of Fraley and Vazire's NF approach (2014) to the clinical psychological literature.

Power is defined as the likelihood that a study can detect a true effect if one exists (Cohen, 1992). Studies are ideally designed to have $80 \%$ power to detect an effect, although in practice they often have lower power due to smaller sample size or a misunderstanding of how statistical significance testing and power are interdependent (Greenland et al., 2016). Work in other subfields has highlighted a glut of potentially false positive findings (e.g., Simmons, Nelson, \& Simonsohn, 2011). Although never desirable, we argue that false positive findings in clinical psychology may lead to even more wasted time, effort, and resources. Adequate power is one of the best ways to minimize the risk for false positives (Marino, 2018). Sample size (N), although only one determinant of statistical power (along with the size of the effect in the population and chosen significance level), has several merits as an index of overall replicability. Indeed, Brunner and Schimmack (2016) have argued that $N$ is a good index of how likely something is to replicate in situations where actual 
replications are impractical or impossible (as is the case with much of the work that clinical psychologists do).

Where subfields such as social and personality psychology often rely on convenience samples, such populations are not ideal for clinical psychology research questions. Clinical psychology typically focuses on low base-rate problems, and often uses necessarily small samples drawn from hard-to-reach populations to study them (e.g., veterans with PTSD, new mothers with postpartum depression). These samples require huge amounts of specialized attention and time devoted to recruitment and screening. We are often making inferences about fragile and complex effects, and may lack appropriate power because of population constraints. This creates the likelihood that much of the research in clinical psychology is underpowered and raises the alarming possibility that we have a replicability problem that urgently needs to be addressed.

Despite the likelihood that clinical psychology faces problems with the robustness of our findings, we may also offer a particular form of expertise in ameliorating this issue. Statisticians have argued that an overreliance on dichotomous decisions thresholds (e.g., $p<.05$ ) has come about because some scientists are not well-equipped to deal with uncertainty and thus use decision thresholds (significant/not significant) to create a false sense of certainty about a nuanced set of results (Cohen, 1994). Clinical psychologists often deal with noisy data and complex patterns of results. We often rely on approaches such as multi-method, multi-informant protocols to try to boost the ratio of signal to noise, even in small samples. Although this does not increase sample size, it can increase reliability, which is relevant for power (i.e., more reliable indicators provide a more powerful test; Humphreys \& Drasgow, 1989; Kanyongo, Brook, Kyei-Blankson, \& Gocmen, 2007). Similarly, clinical psychologists are often faced with challenges inherent in pooling data from different samples, regions, labs, or eras. Despite the difficulties of collecting large samples in clinical psychological research, because of a relatively greater focus on practices that maximize power, we 
predict that clinical psychology will have larger median sample sizes than social psychology. Further, these data pooling and multi-informant approaches may represent a critical component of proposed solutions to increasing power throughout psychology broadly.

Another important way in which clinical psychology is markedly different from social psychology, where issues of replicability have received more attention, is the relative emphasis on correlational versus experimental research designs. Greater relative focus in clinical psychology has been on effect size estimation in correlational designs (e.g., the behavioral correlates of aggression, which symptoms of a disorder may be comorbid with one another). However, experimental approaches are used as well (e.g., determining if a treatment has its intended effect), which provides an opportunity to compare methodological traditions across approaches (Tackett et al. 2017).. To illustrate median power in clinical psychology, we selected two journals: one representing basic research and theory on the broad field of psychopathology (Journal of Abnormal Psychology; JAP) and the other representing treatment and prevention research Journal of Consulting and Clinical Psychology; JCCP). In this way, we hoped to capture both correlational and experimental study designs. While the sample size that confers adequate power does not necessarily differ as a function of study type, it is reasonable to think that experimental studies may involve smaller samples because they are more difficult and costly to conduct, and because they are more often longitudinal (providing more opportunities for attrition). In addition, correlational studies in clinical psychology may be more likely to aim for large samples in order to increase variance in low-base rate symptoms and cases that may fall below threshold for full-blown diagnosis Given this, we expected that studies in JAP would have a higher median power than JCCP, and that differences in median power could be attributed to differences in the predominant study design in each journal. However, we also compared correlational studies to experimental studies directly to determine whether this attribution is a reasonable one. 
Clinical psychologists need to keep up with the replicability conversation to be able to vouch for the robustness of our findings. Critical self-examination provides information about where to start tackling these complex issues. Initiatives proposed in other subfields simply may not be feasible; we need to create our own solutions to address the potential problem of underpowered studies from the bottom up., To make the greatest impact on reducing the burden of mental illness, we need to build a solid foundation of evidence-based recommendations while guarding against outdated scientific practices. Careful examination of power in published clinical psychology studies is an important first step.

\section{Present Study}

Using the methods described in Fraley and Vazire (2014), this study assessed the average statistical power in published studies in JAP and JCCP by calculating the NF. As in Fraley and Vazire (2014), we operationalized NF for these journals as the median sample size of all the studies published within that selected year. To examine trends in NF over time, we selected all journal issues from four years $(2000,2005,2010,2015)$, and coded every article within these issues. The present study pre-registered hypotheses to adhere to the principles of open and transparent research practices. While this work is largely descriptive and we did not have specific quantitative predictions, we thought it was important to transparently account for theoretical priors before engaging in any coding or analyses. Registration materials for this study can be found here: https://osf.io/u43e7/. Hypotheses for the present study were:

1. Clinical/psychopathology journals will have a higher NF than social journals.

2. JAP will have a higher NF than JCCP.

3. Differences in NF may be accounted for by distinctions between correlational and experimental methodology.

\section{Method}


In total, we coded 755 studies in 732 articles (16 articles included more than one study). Two coders recorded the sample size of each study by reading the article, and in cases with discrepancies between coders, the first author made a final decision by consulting the original article. The coders, who were undergraduate psychology students, also made a judgment about whether the study design was experimental or correlational in nature. There were several instances of non-typical studies; we followed the decision rules created by Fraley and Vazire (2014) where applicable and created decision rules for instances unique to clinical papers. In studies with twins, we recorded the number of twin pairs. In treatment studies, the meaningful unit of analysis was sometimes type of therapy, sometimes therapy pairings, and sometimes therapists as participants; we used whichever $N$ was the focal one used for analyses. For longitudinal data, we used the $N$ of the largest wave (usually the initial wave), unless focal analyses depended on participants having more than one wave of data (e.g., change scores), in which case we used the $N$ that met that specified threshold. To deal with skewness caused by some very large sample sizes, we used the median sample size for each year (rather than the mean). To maintain consistency with Fraley and Vazire (2014) and the previous literature, we alternately coded any study that had 1000 or more participants as 999 and examined the mean as an alternative way to deal with skewness.

Again, following Fraley and Vazire (2014), we included all participants as eligible from whom some data was collected. This means that if there was an initial screener, everyone who passed was included in the study $N$, even if some participants were subsequently excluded due to failure to meet diagnostic criteria, or for some other reason (not attending a minimum number of treatment sessions, presence of comorbid diagnoses, etc.). In cases where initial recruitment was conducted based on belonging to some population (e.g., residence in a homeless shelter, receiving treatment for breast cancer), we used as the $N$ the number of participants who consented to participate, as no data was collected before that point. Because of the greater importance of selection criteria in some 
clinical psychological research compared to personality or social psychology studies, there were several instances where the number of cases that passed the initial screener $(\operatorname{coded} N)$ was much larger than the $N$ that was ultimately included in the final analyses for the published paper (analyses N). Given this clinical-specific deviation from Fraley and Vazire (2014), we conducted the analyses again with that analyses $N$ from year 2015 data to see if this substantially impacted our conclusions.

\section{Results}

Table 1 summarizes the NFs for the journals we coded, organized by journal and year. A linear model suggested that the typical sample size of JAP increased by about 9.14 cases per sampled year (Figure 1). JCCP had a higher NF than JAP across the first three sampled years. In 2015, JAP's NF surpassed JCCP's. In addition, across both journals, 96 articles (13.11\% of all articles coded) included a sample that was 1000 participants or larger. For all years, the mean sample size was also calculated when coding $N$ as 999 for large samples (see Table 1). To capture average median sample size for each journal across the four selected years, an average NF score (NF-A) was calculated. JCCP had a slightly higher NF-A (182) compared with JAP (175). NFs for three additional journals: Journal of Personality (JP), Personality and Social Psychology Bulletin (PSPB), and Psychological Science (PS), included in Fraley and Vazire (2014), were selected for comparison purposes to NF scores for the year 2010. Both JAP (164) and JCCP (194) had higher 2010 NFs compared to PSPB (89.5) and PS (76). JCCP's 2010 NF (194) was also higher than JP's 2010 NF (173). A similar pattern was observed when comparing the NF-5 (average median across the five years examined) reported in Fraley and Vazire's (2014) manuscript to the NF-A.

To understand whether the reduction in sample size that frequently occurs as a result of more stringent selection criteria in clinical research impacted our conclusions, we calculated our results for the year 2015 using the analyses $N$ to compare them to the above results obtained using the coded $N$. Specifically, each journal had 39 studies (78 studies overall) where the analyses $N$ was 
smaller than the coded $N$. In JCCP, the analyses $N$ was smaller than the coded $N$ by an average of 106 participants (median difference $=39$ ). In JAP, the analyses $N$ was smaller than the coded $N$ by an average of 115 participants (median difference $=30$ ). Using the analyses $N$ to calculate the $\mathrm{NF}$ for 2015 results in a 2015 NF of 165 for JCCP and 233 for JAP. In both cases, these more conservative estimates of the NF using the analyses $N$ remained within the range of $\mathrm{NF}$ values for that journal overall. However, it is important to note that $41 \%$ of studies were affected (78 out of 190 studies), and thus the present estimates based on the coded $N$ are likely an overestimate of the power of the final analyses for many of these papers.

In line with Fraley and Vazire (2014), we calculated the statistical power of the typical study in clinical journals to detect a range of effect sizes $(r=.10, r=.20, r=.30, r=.40$, and $r=.50)$, but focus our discussion on $r=.20$. This is primarily for ease of comparison to social/personality psychology, but also because .20 has been suggested as a meaningful guideline for clinically relevant effects (Ferguson, 2009). Specifically, in our power calculations, we used $\alpha=.05$ and Ns from Table 1. Both clinical journals had slightly lower than adequate power $(80 \%$; per Cohen, 1992$)$ to detect an effect size of $r=.20$ (JAP: 76\% and JCCP: 78\%; see Table 2). Across years, there was greater variability for estimated power in JAP (ranging from 50\% in 2000 to $92 \%$ in 2015) than JCCP (ranging from $72 \%$ in 2000 to $80 \%$ in 2005 and 2010). Compared to social and personality journals examined by Fraley and Vazire (2014), the power of these two clinical journals was most similar to the most highly powered personality journal, JP (77\%).

Table 3 summarizes the percentages of published articles that were correlational versus experimental organized by journal and year. Most studies published in JAP were correlational (ranging from 91-97.5\% across years). JCCP published fewer correlational studies (ranging from 27$67.5 \%$ across years) and had a greater focus on experimental studies. In addition to using the journals as proxies for study type, we also calculated the median sample size for all correlational 
versus experimental studies within each year and the average median across the four years (Table 3).

On average, correlational studies have a larger median sample size across all years examined, as predicted. Thus, the larger NF for JAP may be explained, at least in part, by the larger proportion of correlational studies in that journal. We estimated the positive rates of findings published in clinical compared to social-personality psychology journals based on their power to detect an effect size of $r$ $=.20$. We created these estimates by using both a $50 \%$ and $80 \%$ probability of the null hypothesis being true. The estimated false-positive rates for $50 \%$ probability of the null hypothesis being true were $6 \%$ (near the nominal 5\% level), whereas the false-positive rates were around $20 \%$ for $80 \%$ probability of the null hypothesis being true. Last, we examined the association between impact factor (IF) and NF of the two clinical journals compared to the social-personality journals that Fraley and Vazire (2014) reported on (see Figure 2). Compared to social-personality journals, the two clinical journals that we examined were both in the upper-right quadrant of the figure, such that higher NF was associated with a higher IF.

\section{Discussion}

The purpose of the present study was to extend Fraley and Vazire's (2014) NF to leading clinical journals, in order to quantify the quality and likely replicability of empirical studies with respect to statistical power. Adequate power is one good indicator of overall trustworthiness because it guards against false positive findings and allows for more precise effect size estimates. Fraley and Vazire (2014) argued that those journals that publish papers with higher NFs should be regarded as more prestigious and credible sources of scientific information. Thus, we calculated the NF for two clinical psychology journals, including one that represents basic research and theory in psychopathology, and the other representing primarily treatment and prevention research, two pillars of research in clinical psychological science. The typical sample size published in these two journals 
across the years 2000, 2005, 2010, and 2015 was 179, with both journals' overall NF very close in size.

Our first hypothesis was supported: clinical journals did, on average, have a higher NF than social/personality journals, which had an overall average sample size of 104 . However, there was much more variation in NF across the six social/personality journals coded by Fraley and Vazire, and the JP, with an NF-5 of 178, was comparable to the two clinical journals. The typical study published in JAP and JCCP had just under 80\% power to detect an effect size of $r=.20$ using $p<$ .05 .

Our second hypothesis, that JAP would have a higher NF than JCCP, was only supported for one of the four years coded, in 2015. For the years 2000, 2005, and 2010, JCCP's NF was larger than JAP's. In 2015, the reverse was true (JAP = 276 and JCCP = 184), which provided the two journals with nearly equivalent NF-As over time (JAP $=175$ and JCCP $=182)$. This difference was hypothesized on the presumption that correlational studies may use larger samples than experimental studies. This was borne out in the present study, which showed that correlational studies in the two clinical journals used larger samples on average than experimental studies $($ correlational average median $=210.75$; experimental average median $=134.88)$. Although we did find evidence for a greater proportion of correlational studies published in JAP than in JCCP, this contrast was not as strong as expected. JCCP published a substantial proportion of both correlational and experimental studies, resulting in less differentiation in overall sample size between the two journals than predicted. In addition, the changing trends over time were not hypothesized, with the most recent issue we coded (2015) supporting our hypothesis (JAP NF > JCCP NF) and evidencing the greatest contrast in correlational vs experimental studies between the two journals. In particular, 2015 also showed the greatest difference between correlational and experimental sample 
sizes, seemingly driven by a large increase in median sample size for correlational studies when compared to previous years (e.g., $2010=194 ; 2015=297$ ).

The false positive rates of findings published in clinical psychology journals were close to the nominal alpha rate of $5 \%$ in situations where there is a $50 \%$ chance of the null hypothesis being true, which are similar to those of higher power social-personality psychology journals such as JP. We examined the statistical power of studies to detect an effect of $r=.20$ primarily for ease of comparison with results from Fraley \& Vazire (2014) and found average power to detect an effect size to be near $80 \%$. When we look at the power to detect an average effect size of $r=.10$, we see a much bleaker picture, with average power to detect an effect size below 30\% (27\% for JCCP and $26 \%$ for JAP). Critically, effect sizes of this magnitude are likely to be relevant for clinical psychologists, as they can still have practical significance when they affect many people or repeatedly affect the same individual (see Greenwald, Banaji, \& Nosek, 2014).

Although the present study used sample size as a proxy for power, $N$ is only one factor that determines a study's power to detect an effect. We did not account for changes in significance level (e.g., reducing alpha to $1 \%$ to account for multiple comparisons); the appropriate use of such familywise Type I error corrections would create the need for even larger samples to maintain the power assumed in the present study. Thus, the present estimates clearly indicate a need for clinical psychology research to increase sample sizes to attain adequate power to detect small effects, which are likely to be most representative of clinical psychology effects of interest.

As previously discussed, it is often difficult and potentially very time consuming to obtain more participants that fit narrowly defined inclusion criteria. This difficulty does not make concerns about power less relevant, however. Thus, clinical psychologists are tasked with appeasing both demands - we need to figure out how to conduct appropriately powered studies while facing the very real constraints imposed by studying limited and often hard to reach populations. Namely, we 
call for more time and energy to be invested in initiatives to pool resources across labs. This may mean thinking carefully about how to harmonize data to compile a large enough sample to be able to draw meaningful statistical inferences if a single lab cannot acquire a sample with the requisite power (e.g., Bakker et al., 2012; Schimmack, 2012).

There are some limitations to the present study. First, as mentioned by Fraley and Vazire (2014), any quantitative measure of journal quality should not be used alone. That is, although we believe the NF provides useful information about the overall state of the published literature in clinical psychology, we do not suggest that a body of research should be judged only by this one metric of research design. Similarly, this method of calculating the NF speaks more directly to the statistical power provided for between-subjects designs and may underestimate power for withinsubjects designs. On the other hand, many of the issues we raised as relevant to clinical samples (e.g., specialized samples, use of multiple informants and multiple methods, pooled data) or relevant to power calculations (e.g., whether 2- or 3-way interactions were used, whether there was any family-wise Type I error control) may mean that these results are an underestimate. Importantly, many clinical studies are likely underpowered for the specific types of analyses they are conducting, but this was not considered as part of our current approach. A critical future direction is a project that more accurately accounts for differences in recommended study design across within- and between-subjects research questions, types of analyses, and more specific sample characteristics (e.g., clinical vs. community samples). Sample size, coded in a global way as it was here, does not provide comprehensive information about power and is only one tool to understand the current state of the field.

In conclusion, the present study examined sample size to shed light on the potential replicability of findings in the published literature in clinical psychology. Overall, two leading clinical psychology journals published studies across a 15-year time span that had slightly inadequate power 
(i.e., $80 \%$ ) to detect a small-moderate effect size $(r=.20)$, and highly inadequate power to detect a small effect size $(r=.10)$. The NFs for JAP and JCCP were comparable, and higher than those estimated for the social/personality journals assessed by Fraley and Vazire (2014). However, we want to caution that this paper likely offers a best-case scenario, as we did not consider correction for multiple comparisons or specific analyses used in in the present study; these considerations would likely produce more conservative estimates. Overall, our findings extend the utility of the NF to clinical psychology journals for the first time, and we hope that these results will motivate focused conversations in the subfield about how to continue to increase study power and ensure the robustness of the clinical psychological literature. 


\section{References}

Bakker, M., van Dijk, A., \& Wicherts, J. M. (2012). The rules of the game called psychological science. Perspectives on Psychological Science, 7, 543-554.

Brunner, J., \& Schimmack, U. (2016). How replicable is psychology? A comparison of four methods of estimating replicability on the basis of test statistics in original studies. retrieved from https://replicationindex.files.wordpress.com/2016/12/psychologicalmethodssubmission.pdf

Chambless, D. L., \& Hollon, S. D. (1998). Defining empirically supported therapies. Journal of Consulting and Clinical Psychology, 66, 7-18.

Chambless, D. L., \& Ollendick, T. H. (2001). Empirically Supported Psychological Interventions: Controversies and Evidence. Annual Review of Psychology, 52, 685-716.

Cohen, J. (1962). The statistical power of abnormal-social psychological research: A review. Journal of Abnormal and Social Psychology, 65.145-153.

Cohen, J. (1992). A power primer. Psychological Bulletin, 112, 155-159.

Cohen, J. (1994). The Earth is round ( $p<.05)$. American Psychologist, 49, 997-1003.

Ferguson, C. J. (2009). An effect size primer: A guide for clinicians and researchers. Professional Psychology: Research and Practice, 40, 532-538.

Fraley, R. C., \& Vazire, S. (2014). The N-Pact Factor: Evaluating the Quality of Empirical Journals with Respect to Sample Size and Statistical Power. PLoS ONE, 9, e109019.

Franco, A., Malhotra, N., \& Simonovits, G. (2014). Publication bias in the social sciences: Unlocking the file drawer. Science, 345, 1502-1505.

Greenland, S., Senn, S. J., Rothman, K. J., Carlin, J. B., Poole, C., Goodman, S. N., \& Altman, D. G. (2016). Statistical tests, P values, confidence intervals, and power: a guide to misinterpretations. European Journal of Epidemiology, 31, 337-350. 
Greenwald, A. G., Banaji, M. R., \& Nosek, B. A. (2014). Statistically small effects of the Implicit Association Test can have societally large effects. Journal of Personality and Social Psychology, 108, $553-561$.

Humphreys, L. G., \& Drasgow, F. (1989). Some comments on the relation between reliability and statistical power. Applied Psychological Measurement, 13, 419-425.

Ioannidis, J. P. A. (2005). Why most published research findings are false. PLoS Medicine, 2, e124.

Kanyongo, G. Y., Brook, G. P., Kyei-Blankson, L., \& Gocmen, G. (2007). Reliability and statistical power: How measurement fallibility affects power and required sample sizes for several parametric and nonparametric statistics. Journal of Modern Applied Statistical Methods, 6, 81-90.

Marino, M. J. (2018). How often should we expect to be wrong? Statistical power, P values, and the expected prevalence of false discoveries. Biochemical Pharmacology, 151, 226-233.

Rossi, J. S. (1990). Statistical power of psychological research: What have we gained in 20 years? Journal of Consulting and Clinical Psychology, 58, 646-656.

Schimmack, U. (2012). The ironic effect of significant results on the credibility of multiple-study articles. Psychological Methods, 17, 551-566.

Simmons, J. P., Nelson, L. D., \& Simonsohn, U. (2011). False-Positive Psychology: Undisclosed Flexibility in Data Collection and Analysis Allows Presenting Anything as Significant. Psychological Science, 22, 1359-1366.

Smaldino, P. E., \& McElreath, R. (2016). The natural selection of bad science. Royal Society Open Science, 3, 160384.

Szucs, D. \& Ioannidis, J. P. A. (2017). Empirical assessment of published effect sizes and power in the recent cognitive neuroscience and psychology literature. PLoS Biology, 153, e2000797 
Tackett, J. L., Lilienfeld, S. O., Patrick, C. J., Johnson, S. L., Krueger, R. F., Miller, J. D., ... Shrout, P. E. (2017). It's Time to Broaden the Replicability Conversation: Thoughts for and From Clinical Psychological Science. Perspectives on Psychological Science, 12, 742-756. 
Table 1

Sample Sizes for JAP and JCCP

\begin{tabular}{lllllll}
\hline & \multicolumn{5}{c}{ Year } \\
\cline { 2 - 7 } & 2000 & 2005 & 2010 & 2015 & NF-A & IF \\
\hline JAP NF & 95 & 165.5 & 164 & 276 & 175.13 & 4.642 \\
JAP mean* & 260.20 & 297.78 & 368.41 & 390.42 & & \\
& & & & & & \\
JCCP NF & 159 & 192 & 194 & 184 & 182.25 & 4.536 \\
JCCP mean* & 307.39 & 354.26 & 352.61 & 314.42 & & \\
& & & & & & \\
JP NF & & & 173 & & 178.1 & 3.483 \\
PSPB NF & & & 89.5 & & 94.6 & 2.498 \\
PS NF & & & 76 & & 72.8 & 6.128
\end{tabular}

Note. JAP = Journal of Abnormal Psychology, NF = N-pact Factor (median), JCCP = Journal of Consulting and Clinical Psychology, JP = Journal of Personality, PSPB = Personality and Social Psychology Bulletin, PS = Psychological Science (social/personality articles only), NF-A = averaged N-pact Factor (median) across the four selected years, IF = impact factor as retrieved from journal's websites on July 8, 2018. $*$ = calculated coding all studies with samples of 1000 or larger as $N=999$. 
Table 2

Power to Detect Effect Sizes in Clinical Compared to Social-Personality Journals

\begin{tabular}{lcccccc}
\hline & & \multicolumn{5}{c}{ Population Effect Size $(r)$} \\
\cline { 3 - 7 } Journal & Year & .10 & .20 & .30 & .40 & .50 \\
\hline$J A P$ & 2000 & .16 & .50 & .85 & .98 & .99 \\
& 2005 & .25 & .74 & .98 & .99 & .99 \\
& 2010 & .25 & .73 & .98 & .99 & .99 \\
& 2015 & .38 & .92 & .99 & .99 & .99 \\
& NF-A & .26 & .76 & .98 & .99 & .99 \\
JCCP & & & & & & \\
& 2000 & .24 & .72 & .97 & .99 & .99 \\
& 2005 & .28 & .80 & .99 & .99 & .99 \\
& 2010 & .28 & .80 & .99 & .99 & .99 \\
& 2015 & .27 & .78 & .99 & .99 & .99 \\
JP & NF-A & .27 & .78 & .99 & .99 & .99 \\
PSPB & & & & & & \\
PS & & .27 & .77 & .98 & .99 & .99 \\
& & .16 & .49 & .84 & .98 & .99 \\
& & .13 & .40 & .74 & .95 & .99 \\
\hline
\end{tabular}

Note. JAP $=$ Journal of Abnormal Psychology, JCCP $=$ Journal of Consulting and Clinical

Psychology, JP = Journal of Personality; PSPB = Personality and Social Psychology Bulletin, PS = Psychological Science (social/personality articles only). NF-A = averaged N-pact Factor across the four selected years.

Power is calculated for a two-tailed test at $\alpha=.05$. 
Table 3

Percentage of Articles that were Correlational versus Experimental by Year and Average Median Sample Size by Study Type

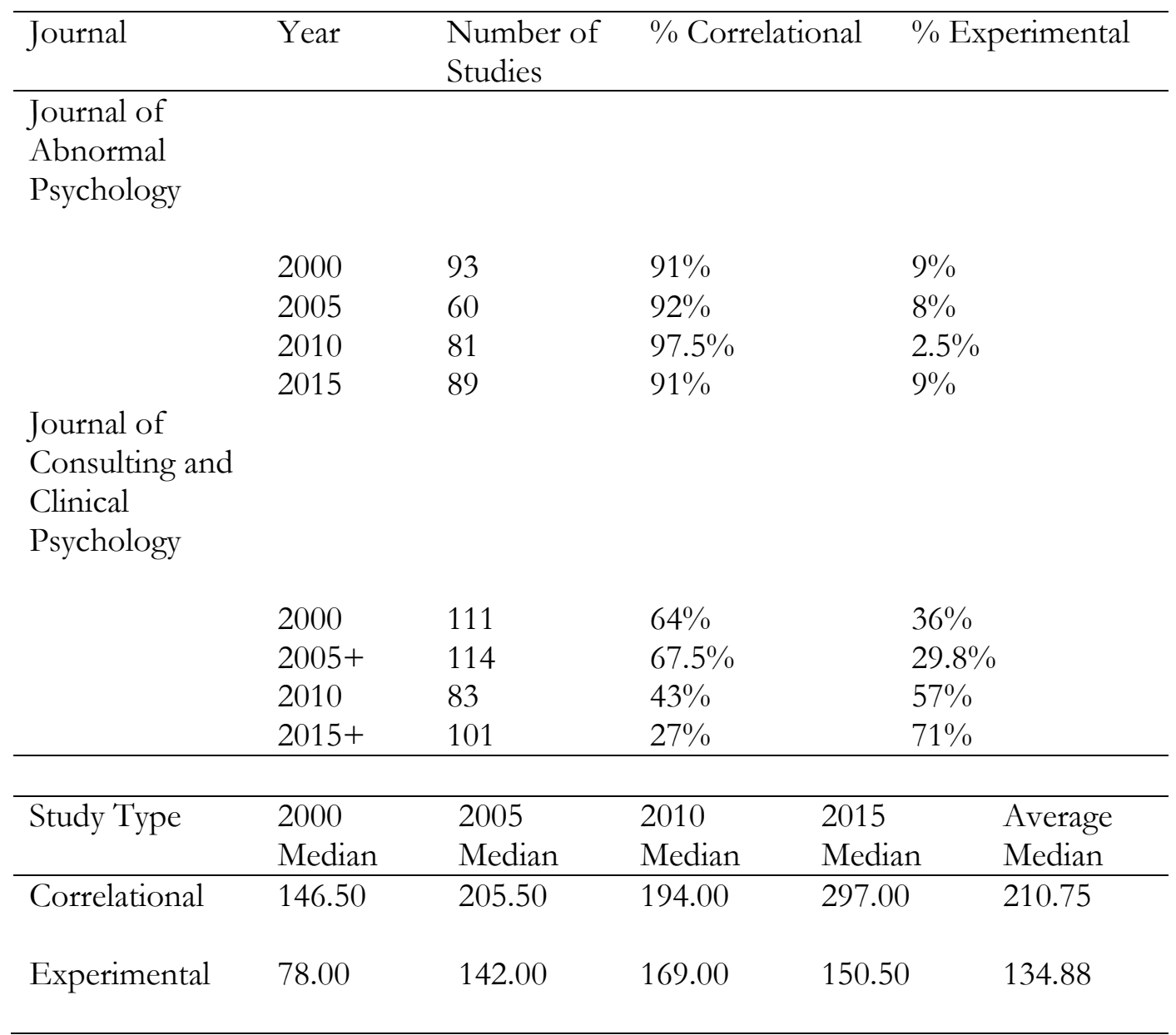

Note. + denotes years in which the journal published articles that were neither correlational nor experimental (i.e., meta-analysis); for these years, the percentages do not add up to $100 \%$. 


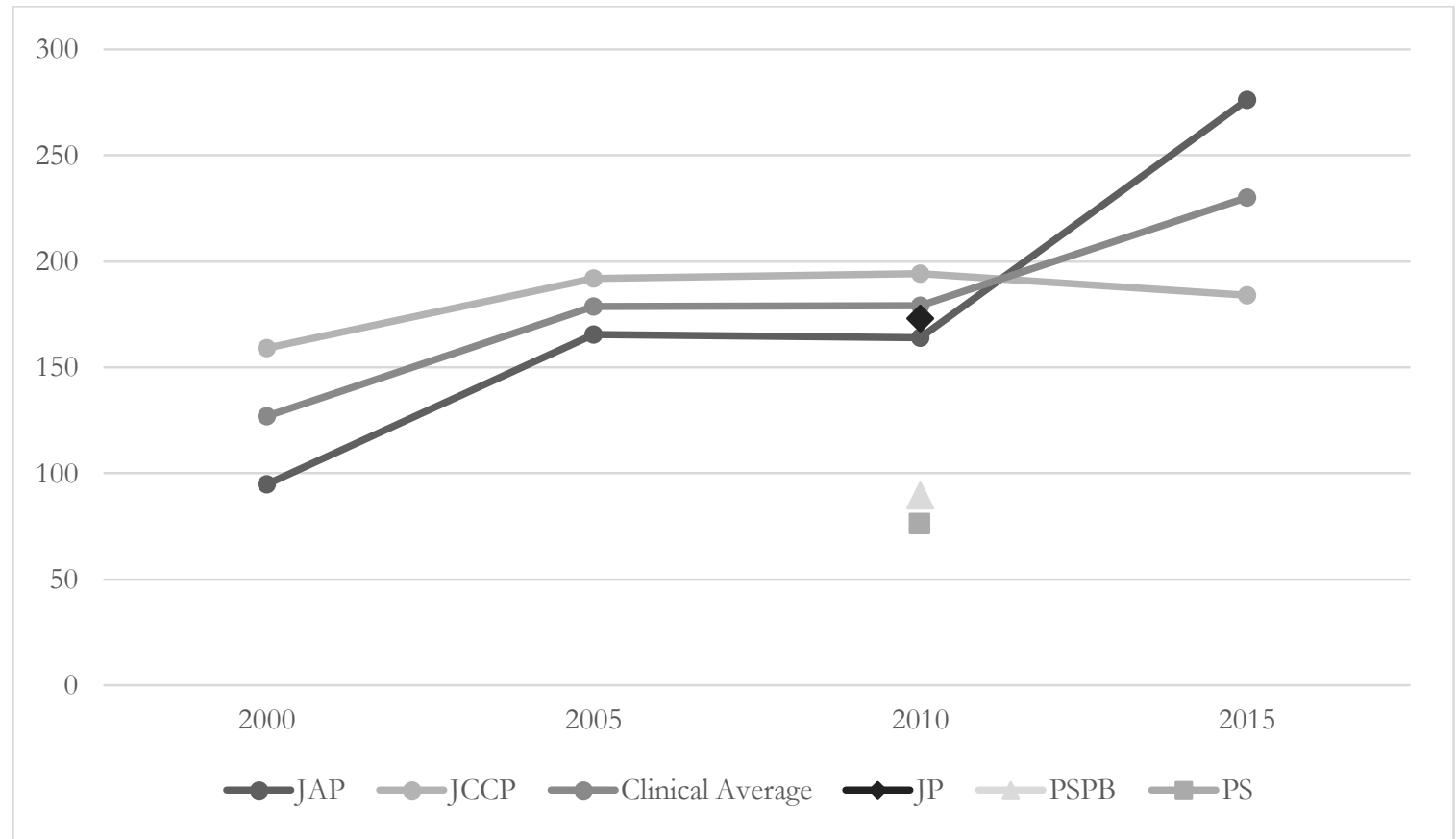

Figure 1. Median Sample Sizes for JAP and JCCP in Clinical Psychology.

Note. JAP $=$ Journal of Abnormal Psychology, JCCP $=$ Journal of Consulting and Clinical Psychology, Clinical Average $=$ arithmetic mean of JAP and JCCP, JP = Journal of Personality; PSPB $=$ Personality and Social Psychology Bulletin, PS = Psychological Science (social/personality articles only). 


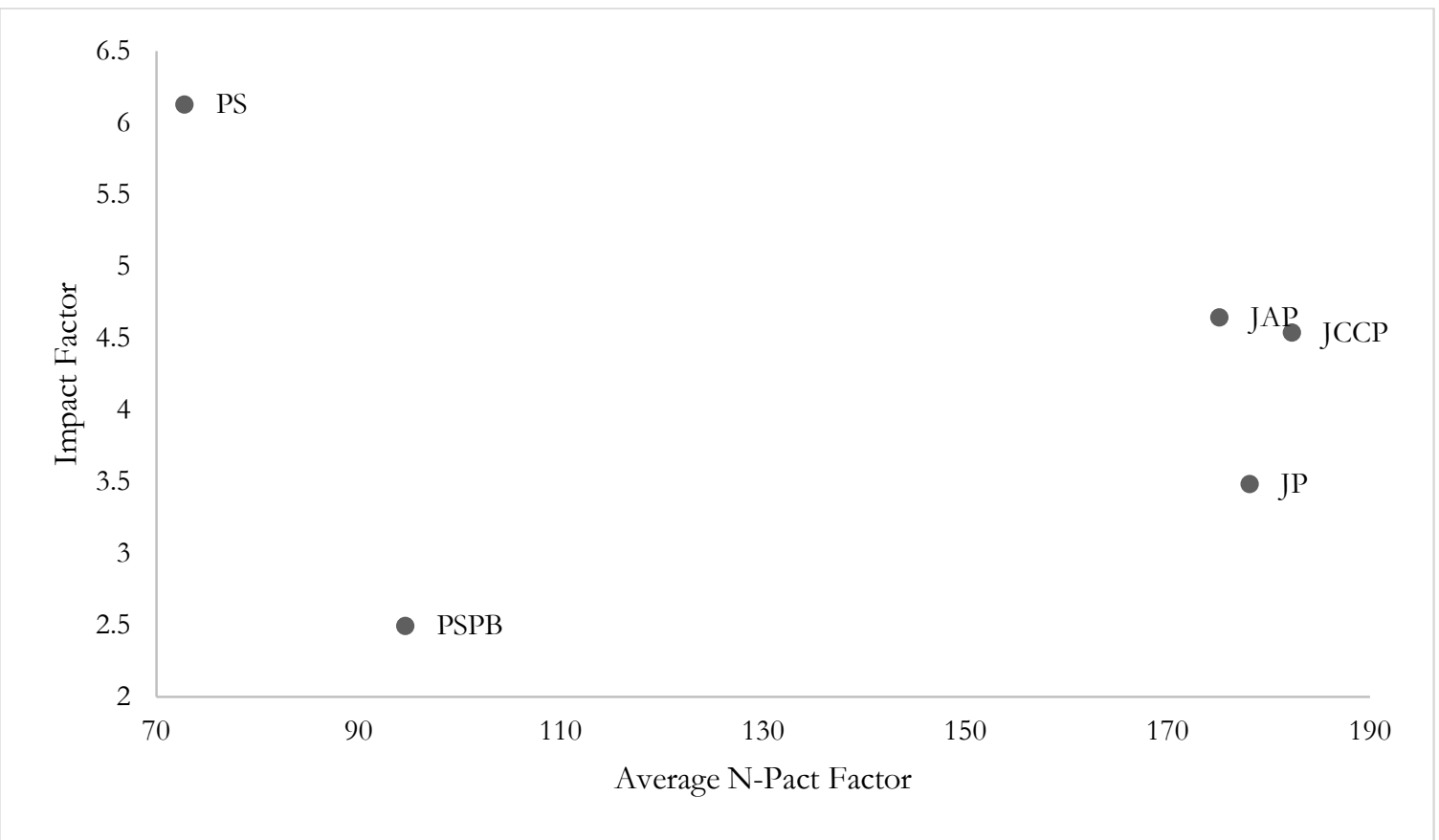

Figure 2. Association between Journals' Average N-Pact Factor (NF-A) and Journal Impact Factor (JIF)

Note. JAP $=$ Journal of Abnormal Psychology, JCCP $=$ Journal of Consulting and Clinical Psychology, JP = Journal of Personality; PSPB = Personality and Social Psychology Bulletin, PS = Psychological Science (social/personality articles only). 